\title{
ESTUDO EPIDEMIOLÓGICO DE 55 PACIENTES PORTADORES DE DOENÇA VERTEBRAL METASTÁTICA SINTOMÁTICA EM SANTO ANDRÉ - SP, BRASIL
}

\author{
EPIDEMIOLOGICAL STUDY OF 55 PATIENTS WITH SYMPTOMATIC METASTATIC \\ SPINAL DISEASE IN SANTO ANDRÉ - SP, BRAZIL
} ESTUDIO EPIDEMIOLÓGICO DE 55 PACIENTES CON ENFERMEDAD METASTÁSICA
SINTOMÁTICA DE LA COLUMNA VERTEBRAL EN SANTO ANDRÉ - SP, BRASIL

Edgar Santiago Valesin Filho ${ }^{1}$, Ricardo Tardini1', Luiz Carlos de Abreu², Bruno Vieira Motter ${ }^{3}$, Fernando Adami ${ }^{4}$, Luciano Miller Reis Rodrigues ${ }^{5}$

\begin{abstract}
RESUMO
Objetivo: Apresentar o perfil epidemiológico dos pacientes portadores de doença vertebral metastática sintomática de serviço público de atenção terciária na região do ABC. Método: Avaliamos de Janeiro de 2008 a Janeiro de 2011, 55 pacientes com diagnóstico de metástase vertebral e coletamos dados de idade no diagnóstico de lesão metastática, sexo, tipo de tumor, topografia vertebral e sintomatologia. A evolução da doença foi observado nas datas: biópsia da neoplasia primária; início de sintomas vertebrais; diagnóstico de doença vertebral metastática (imagem) e data da cirurgia. Resultados: A idade dos pacientes variou de 28 a 85 anos; $40 \%$ homens e $60 \%$ mulheres. Os principais tumores foram carcinoma de mama (32,7\%), mieloma múltiplo $(25,4 \%)$ e carcinoma de próstata (14,5\%). Observamos 25 pacientes (45,4\%) com lesões na coluna torácica; 13 pacientes (23,6\%) lombares ou sacrais; 11 pacientes (20\%) difusas e 6 pacientes $(10,9 \%)$ cervicais. 34 pacientes $(61,8 \%)$ apresentavam somente dor, os demais $(38,2 \%)$ apresentavam também alteração neurológica. 0 intervalo entre a lesão primária e a manifestação clínica de lesão vertebral apresentou mediana de 190 dias; entre a sintomatologia na coluna e o diagnóstico por imagem teve mediana de 70 dias; aos submetidos a cirurgia, entre o diagnóstico e o procedimento foi de 288 dias. Conclusão: Observamos os dados epidemiológicas, compatíveis a literatura: predomínio do sexo feminino (60\%), com idade média de 55 anos; metástases predominantemente toracolombares (69\%) por neoplasia de mama, mieloma múltiplo e próstata (72\%). Observamos ampla variação no intervalo de tempo na descrição cronológica dos eventos clínico-diagnósticos e cirúrgicos.
\end{abstract}

Descritores: Coluna vertebral; Metástase neoplásica; Neoplasias da medula espinal; Qualidade de vida; Descompressão.

\begin{abstract}
Objective: To present the epidemiological profile of patients with symptomatic metastatic spinal disease treated in tertiary public care in the ABC region. Method: We evaluated 55 patients with vertebral metastases and collected data on age at diagnosis of metastatic lesion, sex, tumor type, spinal topography and symptoms from January 2008 to January 2011. Disease progression was observed on the following occasions: biopsy of the primary tumor, early spinal symptoms, diagnosis of spinal metastatic disease (images), and date of surgery. Results: The patients' ages ranged from 28 to 85 years; 40\% were men and 60\% were women. The primary tumors were breast carcinoma (32.7\%), multiple myeloma (25.4\%), and prostate carcinoma (14.5\%). We observed 25 patients (45.4\%) with lesions in the thoracic spine, 13 patients (23.6\%) with lumbar or sacral lesions, 11 patients (20\%) with diffuse lesions, and 6 patients (10.9\%) with cervical lesion. Thirty four patients (61.8\%) had only pain, and the others (38.2\%) had also neurological changes. The median intervals between the primary lesion and the clinical manifestation of vertebral lesion was 190 days; between the spine symptoms and the imaging diagnosis the median was 70 days; for those who underwent surgery, the median between the diagnosis and the procedure was 288 days. Conclusion: The epidemiological data were consistent with the literature: female predominance (60\%), mean age was 55 years; thoracolumbar metastases were predominantly (69\%) due to breast cancer, multiple myeloma and prostate cancer (72\%). We observed wide variation in the interval between the chronological description of clinical events, both diagnostic and surgical.
\end{abstract}

Keywords: Spine; Neoplasm metastasis; Spinal cord neoplasms; Quality of life; Decompression.

\section{RESUMEN}

Objetivo: Presentar el perfil epidemiológico de los pacientes con síntomas de enfermedad metastásica espinal tratados en el servicio público de atención terciaria en la región del ABC. Método: Se evaluaron 55 pacientes con diagnóstico de metástasis vertebral y se recogieron datos sobre la edad al diagnóstico de la lesión metastásica, sexo, tipo de tumor, la topografía y los síntomas espinales de enero 2008 a enero 2011. La progresión de la enfermedad se observó en las siguientes ocasiones: biopsia del tumor primario, primeros síntomas de la columna vertebral, diagnóstico de la enfermedad metastásica espinal (imágenes) y fecha de la cirugía. Resultados: La edad de los pacientes osciló entre 28 y 85 años; el 40\% eran hombres y el 60\% mujeres. Los tumores primarios fueron carcinoma de mama (32,7\%), mieloma múltiple (25,4\%) y carcinoma de próstata (14,5\%). Hemos observado 25 pacientes (45,4\%) con lesiones en la columna vertebral torácica, 13 pacientes $(23,6 \%)$ con lesiones lumbares o sacras, 11 pacientes (20\%) con lesiones difusas y 6 pacientes (10,9\%) con lesiones cervicales. Treinta y cuatro pacientes $(61,8 \%)$ tenían sólo dolor y el restante $(38,2 \%)$ también tenía trastornos neurológicos. Los intervalos promedio entre la lesión primaria y la manifestación clínica de la lesión vertebral fue de 190 días, entre los síntomas de columna y el diagnóstico por

1. Médico Ortopedista, Assistente do Grupo de Coluna da Faculdade de Medicina do ABC - Santo André, SP, Brasil.

2. Professor do Departamento de Saúde da Coletividade da Faculdade de Medicina do ABC - Santo André, SP, Brasil.

3. Médico Residente em Ortopedia eTraumatologia (R3) pela da Faculdade de Medicina do ABC - Santo André, SP, Brasil.

4. Professor do Laboratório de Escrita Científica da Faculdade de Medicina do ABC - Santo André, SP, Brasil.

5. Médico Ortopedista, Chefe do Grupo de Coluna da Faculdade de Medicina do ABC - Santo André, SP, Brasil.

Trabalho realizado no Hospital Estadual Mario Covas de Santo André, Santo André, SP, Brasil.

Correspondência: Ricardo Tardini. Rua Santo André, 678, CEP 09020-230 - Santo André, SP, Brasil. rtardini@uol.com.br

Recebido em 06/12/2012, aceito em 08/01/2013. 
imágenes la mediana fue de 70 días; para aquellos que se sometieron a la cirugía, la mediana entre el diagnóstico y el procedimiento fue de 288 días. Conclusión: Los datos epidemiológicos fueron compatibles con la literatura: predominio del sexo femenino (60\%), edad media de 55 años, predominantemente metástasis toracolumbar (69\%) debido a cáncer de mama, mieloma múltiple y cáncer de próstata (72\%). Se observó una amplia variación en el intervalo entre la descripción cronológica de eventos clínicos ya sean diagnósticos o quirúrgicos.

Descriptores: Columna vertebral; Metástasis de la Neoplasia; Neoplasias de la médula espinal; Calidad de vida; Descompresión.

\section{INTRODUÇÃO}

A evolução das terapias oncológicas associada ao diagnóstico precoce tem proporcionado maior sobrevida aos pacientes acometidos por lesões metastáticas. Segundo a literatura, mais de $10 \%$ dos pacientes com câncer irão desenvolver doença vertebral secundária sintomática, com envolvimento de múltiplos níveis em mais de 40 a $70 \%$ dos $\operatorname{casos}^{1-3}$. Assim, a doença vertebral metastática (DVM) é uma afecção que tem se apresentado com frequência e caracteriza-se por representar uma das principais complicações do tratamento ao decorrer da evolução de pacientes oncológicos, causando dor e comprometendo de maneira significativa sua qualidade de vida ${ }^{4-7}$.

Aproximadamente 1,4 milhão de pacientes são anualmente diagnosticados com câncer nos EUA ${ }^{8}$, sendo o esqueleto axial reconhecidamente o sítio mais frequente de metástase óssea em pacientes com doença neoplásica sistêmica9 ${ }^{9}$. A maior parte dos tumores da coluna vertebral são metástases, com aproximadamente 18.000 a 25.000 novos casos diagnosticados nos EUA anualmente ${ }^{10,11}$. As metástases para a coluna vertebral podem ocorrer em três situações anatômicas: extramedular, intraduralextramedular ou intramedular; entretanto mais de 98\% das lesões ocorrem na região extradural, em virtude do efeito de barreira promovido através da dura-máter, dificultando a progressão da lesão para a medula ${ }^{12,13}$. A população frequentemente acometida, segundo a literatura, é caracterizada por equilíbrio na distribuição de sexo, com alguns artigos demonstrando leve superioridade em mulheres, sendo o tumor de mama, acometendo a região toracolombar o mais prevalente $e^{4,13,14}$

Assim, o objetivo do presente estudo é demonstrar os dados epidemiológicos apresentados em uma população portadora de doença vertebral metastática em Santo André, SP, Brasil, em relação ao sexo, idade, topografia da lesão na coluna vertebral, tipo de neoplasia, e sintomatologia principal. Buscamos também descrever eventos clínicos e diagnósticos observados de maneira cronológica durante a evolução destes pacientes.

\section{MATERIAL E MÉTODO}

Estudo retrospectivo de 55 pacientes avaliados em equipe de ortopedia especializada em cirurgia da coluna vertebral em um mesmo serviço, no Hospital Estadual Mario Covas, em Santo André, SP, Brasil. Os dados foram coletados durante o período de Janeiro de 2008 a Janeiro de 2011, em avaliações ambulatoriais e hospitalares de pacientes com diagnóstico de metástase em coluna vertebral, apresentando sintomatologia compatível com a lesão apresentada em exames de imagem correspondentes. Observamos também através de prontuários médicos as seguintes informações: sexo, idade no diagnóstico confirmatório de lesão metastática, tipo de tumor, topografia (cervical, torácica, lombossacral ou difusa, ao acometer mais de uma das regiões anteriores concomitantemente) e sintomatologia principal (dor, alteração neurológica ou ambas condições). Apresentamos dados cronológicos da evolução da doença, com os seguintes momentos de observação: 1) data de biópsia referente à neoplasia primária; 2) data de início de sintomas vertebrais; 3) data de diagnóstico confirmatório de doença vertebral metastática através de exame de imagem (RM); 4) data de cirurgia, para os pacientes que foram submetidos à descompressão e estabilização segmentar.

Todas as lesões descritas apresentaram-se sintomáticas e foram encaminhadas para avaliação ortopédica especializada após atendimento em equipe multidisciplinar de oncologia. Foram submetidos ao tratamento cirúrgico 30 pacientes $(54,54 \%)$ e 25 pacientes $(45,45 \%)$ foram tratados através de radioterapia e demais medidas clínico-oncológicas.

\section{RESULTADOS}

A idade média dos pacientes variou de 28 a 85 anos, com média de 55,92 anos; houve 22 homens (40\%) e 33 mulheres (60\%). Os principais tumores foram: 18 casos de carcinoma de mama (32,7\%), 14 casos de mieloma múltiplo $(25,4 \%), 8$ casos de carcinoma de próstata (14,5\%), 3 casos de carcinoma gástrico (5,4\%), 2 casos de neoplasia de pulmão (3,6\%), 2 casos de neoplasia renal (3,6\%) e 8 casos de outros tumores (14,5\%) (Figura 1).

Observamos 25 pacientes $(45,4 \%)$ com lesões na coluna torácica; 13 pacientes (23,6\%) com lesões lombares ou sacrais; 11 pacientes (20\%) com lesões difusas e 6 pacientes $(10,9 \%)$ com lesões cervicais (Figura 2).

No momento do diagnóstico confirmatório de lesão vertebral, 34 pacientes $(61,8 \%)$ apresentavam somente dor, sendo que os demais 21 pacientes $(38,2 \%)$ apresentavam dor e alteração neurológica simultaneamente no exame clínico. Foram submetidos ao tratamento cirúrgico 30 pacientes $(54,54 \%)$ e 25 pacientes $(45,45 \%)$ foram tratados através de radioterapia e demais medidas clínico-oncológicas.

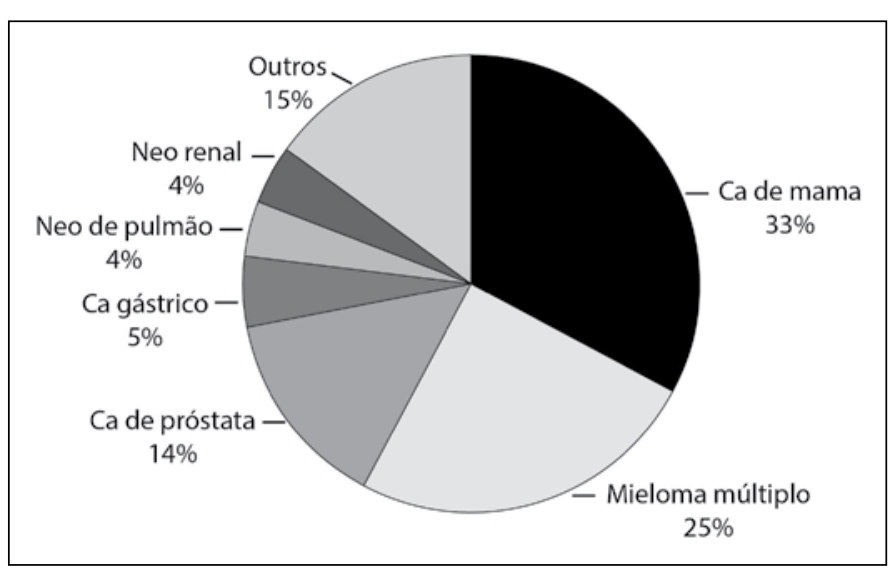

Figura 1. Distribuição percentual dos tipos de tumores primários encontrados nos 55 pacientes com metástases vertebrais.

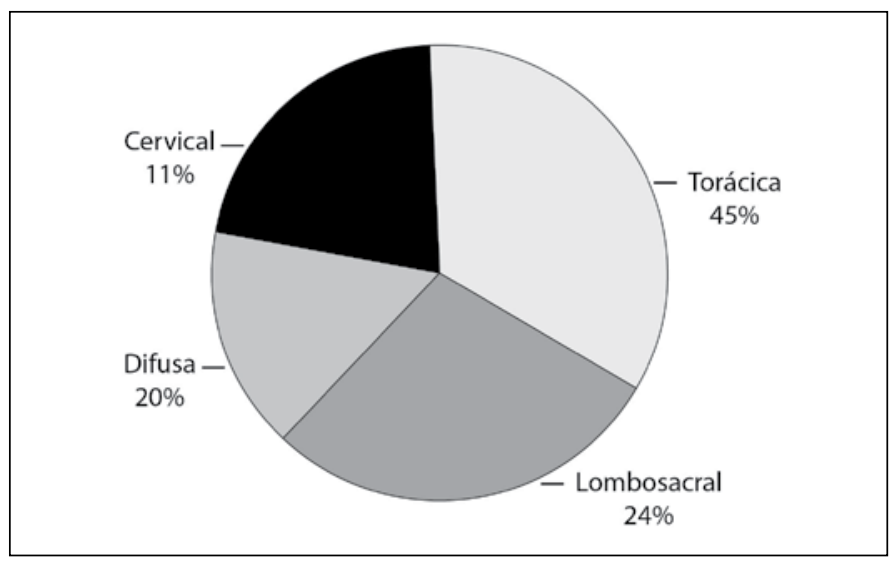

Figura 2. Distribuição percentual do local de acometimento vertebral da lesão metastática. 


\section{ANÁLISE ESTATIISTICA}

Os dados de tempo (t1, t2, t3 e t4) foram descritos por meio de valores de mediana, mínimo, máximo e percentis 25 e 75, uma vez que não apresentaram distribuição normal pelo teste de Shapiro-Wilk $(\mathrm{p}<0,05)$. Utilizou-se o programa estatístico Stata 11.0 para as análises (Tabela 1).

O intervalo entre o diagnóstico da lesão primária e a manifestação clínica inicial de lesão vertebral (t1) apresentou mediana de 190 dias (percentil $25=-61$, percentil $75=756$ ), sendo os valores mínimo e máximo -437 e 3011 dias respectivamente. O intervalo entre a sintomatologia em coluna e o diagnóstico confirmatório (t2) da presença de lesão metastática por imagem (ressonância magnética) apresentou mediana de 70 dias (percentil 25=25, percentil $75=195)$, sendo os valores mínimo e máximo -764 e 1742 dias respectivamente. Em relação aos pacientes que foram submetidos ao tratamento cirúrgico, o intervalo médio entre o diagnóstico confirmatório e o procedimento cirúrgico(t3) apresentou mediana de 128,5 dias (percentil $25=65$, percentil $75=226$ ), sendo os valores mínimo e máximo 5 e 485 dias respectivamente. O intervalo entre o diagnóstico de tumor primário e a cirurgia para tratamento de metástase vertebral (t4) para os 30 pacientes submetidos ao procedimento apresentou mediana de 288 dias (percentil $25=84$, percentil $75=756$ ), sendo os valores mínimo e máximo 4 e 3183 dias respectivamente. A medida de tempo relacionada ao intervalo t1 em seu percentil 25, bem como os valores mínimos de t1 e t2 são demonstradas em números negativos; uma vez que, para alguns pacientes, o sintoma vertebral precedeu o diagnóstico de tumor primário (t1 negativo); da mesma forma, os valores no intervalo t2 em que o diagnóstico de metástase vertebral precedeu a sua sintomatologia, ou seja, foram "achados" em exames de imagem, são também expressos através de números negativos.

Tabela 1. Medidas de tendência central e de dispersão dos tempos t1, t2, t3 e t4 referentes a pacientes portadores de doença vertebral metastática sintomática.

\begin{tabular}{c|c|c}
\hline Medida de tempo (dias) & $\begin{array}{c}\text { Mediana } \\
\text { (Percentil 25; Percentil 75) }\end{array}$ & Mínimo; Máximo \\
\hline t1 $(n=55)$ & $190(-61 ; 756)$ & $-437 ; 3011$ \\
\hline t2 $(n=55)$ & $70(25 ; 195)$ & $-764 ; 1742$ \\
\hline t3 $(n=30)$ & $128,5(65 ; 226)$ & $5 ; 485$ \\
\hline t4 $(n=30)$ & $288(84 ; 756)$ & $4 ; 3183$ \\
\hline
\end{tabular}

t1: Tempo decorrido entre a biópsia do sítio primário até início dos sintomas vertebrais.

t2: Tempo decorrido entre início dos sintomas vertebrais até diagnostico confirmatório do tumor vertebral. t3: Tempo decorrido entre o início dos sintomas vertebrais até cirurgia vertebral.

t4: Tempo decorrido entre a biópsia do sítio primário até cirurgia vertebral.

\section{DISCUSSÃO}

A idade média dos pacientes observados no presente estudo variou de 28 a 85 anos, com média de 55,92 anos; houve 22 homens (40\%) e 33 mulheres (60\%). Segundo a literatura, sexo masculino e feminino são acometidos de maneira semelhante, com leve predomínio do sexo feminino em alguns relatos ${ }^{4,13,15}$ entretanto não parece haver predomínio significativo de sexo nesta afecção. North et al. ${ }^{16}$ identificaram em uma população de 61 pacientes portadores de DVM a idade média de 52,4 anos; Ibrahim et al. ${ }^{14}$ encontraram idade média de 61 anos entre 223 pacientes acometidos por DVM. No presente estudo, podemos atribuir o predomínio de pacientes do sexo feminino em virtude do elevado índice de neoplasia de mama em tratamento no serviço de oncologia, referência para esta afecção na região.

Em estudo envolvendo 842 autópsias consecutivas de pacientes oncológicos sabidamente portadores de doença metastática, Ortiz Gomez ${ }^{17}$ demonstrou que $65 \%$ dos casos de DVVM ocorreram em decorrência de lesões primárias de mama, pulmão, mieloma múltiplo e linfomas. Demais estudos comprovam a incidência principalmente de neoplasia primária de mama, pulmão, próstata, rins, mieloma e linfomas como principais responsáveis por DVM ${ }^{14,18-20}$.
Encontramos a seguinte distribuição etiológica: 18 casos de carcinoma de mama (32,7\%), 14 casos de mieloma múltiplo (25,4\%), 8 casos de carcinoma de próstata (14,5\%), 3 casos de carcinoma gástrico (5,4\%), 2 casos de neoplasia de pulmão (3,6\%), 2 casos de neoplasia renal $(3,6 \%)$ e 8 casos de outros tumores (14,5\%), As informações são compatíveis com a literatura; o tumor de mama parece ser um dos mais frequentes nos casos de DVM, colocando-se à frente em incidência em relação aos demais tumores ${ }^{16}$.

Segundo a literatura, as metástases vertebrais ocorrem mais frequentemente na região lombar, com $70 \%$ dos casos; em seguida na região torácica, com $20 \%$ e por fim, as lesões cervicais, que representam aproximadamente $10 \%$ dos casos de DVM ${ }^{15,19,21,22}$. Entretanto, as lesões torácicas são as mais sintomáticas, representando aproximadamente $70 \%$ dos casos, em virtude de um menor espaço disponível para a medula espinhal nesta região, uma vez que o canal vertebral é caracteristicamente mais estreito ${ }^{1,22}$. Através de estudos realizados em cadáveres, estima-se que a frequência de lesão vertebral em pacientes que morrem devido à neoplasia maligna, varie de 30 a $90 \%{ }^{23,24}$. Usualmente muitos pacientes em tratamento oncológico apresentam lesões assintomáticas ou demais outras complicações graves decorrentes da evolução do quadro neoplásico que não permitem o diagnóstico preciso para exata caracterização das metástases; assim, os valores totais de lesões e sua respectiva distribuição topográfica torna-se um viés.Observamos no presente estudo 25 pacientes $(45,4 \%)$ com lesões na coluna torácica; 13 pacientes $(23,6 \%)$ com lesões lombares ou sacrais; 11 pacientes com lesões difusas e seis pacientes com lesões cervicais (Figura 2).

A sintomatologia principal da lesão vertebral metastática é a dor, caracteristicamente intensa, com piora noturna e à mobilização axial. Radiculopatia, fratura patológica, mielopatia e deformidades progressivas são frequentes. Para alguns autores,dor axial de início súbito em pacientes oncológicos deve ser tratada como indicador de DVM, até que exames documentem o contrário ${ }^{13,25}$. Segundo a literatura ${ }^{25-28}$ aproximadamente $90 \%$ dos pacientes com DVM apresentam dor e em $47 \%$ a $76 \%$ dos casos ocorre alteração neurológica ${ }^{29,30}$; em nosso estudo observamos no momento do diagnóstico confirmatório que, 34 pacientes $(61,8 \%)$ apresentavam somente dor, sendo que os demais 21 pacientes $(38,2 \%)$ apresentavam dor e alteração neurológica simultaneamente no exame clínico.

Observamos que em $32,72 \%$ dos casos, o sintoma vertebral foi prévio ao diagnóstico primário, ou seja; nestes casos, a DVM representou a manifestação clínica inicial da neoplasia primária; segundo a literatura, 10 a 20\% dos pacientes com câncer apresentam sintomatologia inicial através da $\mathrm{DVM}^{31-33}$.

Segundo North et al. ${ }^{16}$, em série de casos acompanhados por DVM, a doença primária foi diagnosticada em média 3,5 anos anteriormente ao diagnóstico secundário em coluna, com variação de 0 a 32 anos. Nesta análise, observamos um intervalo em dias que apresentou mediana (t1) de 190 dias; aproximadamente 6 meses (190 dias) desde o diagnóstico de neoplasia primária até o início da sintomatologia da DVM, embora o tempo máximo observado foi de aproximadamente 8,2 anos. Segundo Levack et al. ${ }^{7}$, deve-se investigar queixas de dores em coluna em pacientes oncológicos com atenção; em estudo envolvendo 319 pacientes com câncer, houve um intervalo tempo médio de 2 meses após o início do quadro álgico em coluna para diagnóstico confirmatório de DVM, considerado um atraso no início do tratamento específico da patologia. Observamos um intervalo que apresentou mediana de 70 dias desde o início dos sintomas vertebrais até o diagnóstico confirmatório, com valor máximo de 1742 dias, aproximadamente 4,7 anos. Entretanto, 6 (10,90\%) pacientes apresentaram diagnóstico de DVM previamente à sintomatologia vertebral, ou seja, nestes casos (valores negativos em t2), o diagnóstico de lesão foi incidental ou um "achado" em exames de imagem direcionados à outros propósitos. $18(32,72 \%)$ pacientes apresentaram diagnóstico de tumor primário após inicio de sintomas vertebrais (valores em t1 negativos); assim, para estes pacientes, o quadro de dor ou alteração neurológica foi a manifestação clínica inicial de uma neoplasia primária, até então oculta. Em relação aos 30 
pacientes que foram submetidos à cirurgia, o intervalo de tempo decorrente desde o diagnóstico confirmatório até o procedimento (t3) apresentou mediana mediana de 128,5 dias (percentil 25 $=65$, percentil $75=226$ ), sendo os valores mínimo e máximo 5 e 485 dias respectivamente. O intervalo que demonstra a progressão da doença neoplásica, desde o diagnóstico do tumor primário até o desfecho cirúrgico da metástase vertebral (t4) para os 30 pacientes submetidos ao procedimento apresentou mediana de 288 dias, ou 9,6 meses (percentil $25=84$, percentil $75=756$ ), sendo os valores mínimo de 4 dias e máximo de aproximadamente 8,72 anos. Devido à ampla margem de valores encontrados na análise, acreditamos que diversos fatores interferiram nestes resultados: neoplasias histologicamente distintas, tratamentos diferentes para cada doença, pacientes em diferentes estágios evolutivos da afecção, complicações que comprometem o tratamento quimioterápi$\mathrm{co}$, entre outros. Assim, entendemos que estudos futuros devem ser direcionados a fatores específicos, como populações restritas à um tipo único de doença primária, podendo assim, possivelmente demonstrar tendências evolutivas relacionadas à agressividade de determinados tipos de neoplasia

Nosso estudo teve como limitação o pequeno número de pacientes avaliados principalmente para dar sustentação a interessante discordância com a literatura em relação ao sintoma de dor e/ou déficit neurológico se apresentar como sintoma primário de dignóstico da doença neoplásica. Com um estudo maior poderemos avaliar com precisão os diferentes tempos.

\section{CONCLUSÃO}

Os resultados obtidos de prevalência em relação ao tipo histológico da neoplasia primária, idade média e o segmento da coluna vertebral acometido pelas metástases estão de acordo com a literatura internacional.

Observamos que a doença vertebral metastática como sintoma (dor e/ou alteração neurológica ) primário de diagnóstico da doença neoplasica foi maior que a média da literatura.

\section{REFERÊNCIAS}

1. Grant R, Papadopoulos SM, Greenberg HS. Metastatic epidural spinal cord compression. Neurol Clin. 1991;9(4):825-41.

2. Heldmann U, Myschetzky PS, Thomsen HS. Frequency of unexpected multifocal metastasis in patients with acute spinal cord compression. Evaluation by low-field MR imaging in cancer patients. Acta Radiol. 1997:38(3):372-5.

3. Sundaresan N, Digiacinto GV, Hughes JE, Cafferty M, Vallejo A. Treatment of neoplastic spinal cord compression: results of a prospective study. Neurosurgery. 1991;29(5):645-50.

4. Rodrigues LMR, Valesin Filho ES, Ueno FH, Fujiki EN, Milani C. Qualidade De Vida De Pacientes Submetidos À Descompressão Por Lesão Vertebral Metastática: Acta Ortop Bras. 2011;19(3):149-5.

5. Tomita K, Kawahara N, Kobayashi T, Yoshida A, Murakami H, Akamaru T. Surgical strategy for spinal metastases. Spine (Phila Pa 1976). 2001;26(3):298-306.

6. Valesin Filho ES, de Abreu LC, Lima GH, de Cubero DI, Ueno FH, Figueiredo GS, et al. Pain and quality of life in patients undergoing radiotherapy for spinal metastatic disease treatment. Int Arch Med. 2013:6(1):6

7. Levack P, Graham J, Collie D, Grant R, Kidd J, Kunkler I, et al. Don't wait for a sensory level-listen to the symptoms: a prospective audit of the delays in diagnosis of malignant cord compression. Clin Oncol (R Coll Radiol). 2002;14(6):472-80.

8. American Cancer Society. Cancer Facts And Figures 2005. Atlanta: American Cancer Society; 2005.

9. Harrington KD. Metastatic disease of the spine. J Bone Joint Surg Am. 1986;68(7):1110-5

10. Gokaslan ZL, York JE, Walsh GL, McCutcheon IE, Lang FF, Putnam JB Jr, et al. Transthoracic vertebrectomy for metastatic spinal tumors. J Neurosurg. 1998;89(4):599-609.

11. Schiff D. Spinal cord compression. Neurol Clin. 2003;21(1):67-86.

12. Jacobs WB, Perrin RG. Evaluation and treatment of spinal metastases: na overview. Neurosurg Focus. 2001;11(6):e10.

13. Perrin RG, Livingston KE, Aarabi B. Intradural extramedullary spinal metastasis. A report of 10 cases. J Neurosurg. 1982;56(6):835-7.

14. Ibrahim A, Crockard A, Antonietti P, Boriani S, Bünger C, Gasbarrini A, et al. Does spinal surgery improve the quality of life for those with extradural (spinal) osseous metastases? An international multicenter prospective observational study of 223 patients. Invited submission from the Joint Section Meeting on Disorders of the Spine and Peripheral Nerves, March 2007. J Neurosurg Spine. 2008;8(3):271-8

15. Mattana JL, Freitas RR, Mello GJP, Armani Neto M, Freitas Filho G, Ferreira CB. Estudo Da Aplicabilidade Do Escore De Tokuhashi Modificado Nos Pacientes Tratados Cirurgicamente De Metástases Vertebrais: Rev Bras Ortop. 2011;46(4):424-30

16. North RB, LaRocca VR, Schwartz J, North CA, Zahurak M, Davis RF, et al. Surgical

management of spinal metastases: analysis of prognostic factors during a 10-year experience. J Neurosurg Spine. 2005;2(5):564-73.

17. Ortiz Gómez JA. The incidence of vertebral body metastases. Int Orthop.1995;19(5):309-11.

18. Oliveira Junior AV, Bortoletto $A$, Rodrigues LCL. Avaliação do tratamento cirúrgico nos pacientes com metástase vertebral secundáriaao carcinoma de mama. Coluna/Columna. 2012;11(3): 226-9.

19. Abdu WA, Provencher M. Primary bone and metastatic tumors of the cervical spine Spine (Phila Pa 1976). 1998:23(24):2767-77.

20. Hammerberg KW. Surgical treatment of metastatic spine disease. Spine (Phila Pa 1976). 1992;17(10):1148-53.

21. Nottebaert M, von Hochstetter AR, Exner GU, Schreiber A. Metastatic carcinoma of the spine. A study of 92 cases. Int Orthop. 1987:11(4):345-8

22. Gokaslan ZL, York JE, Walsh GL, McCutcheon IE, Lang FF, Putnam JB Jr,et al. Transthoracic vertebrectomy for metastatic spinal tumors. J Neurosurg. 1998;89(4):599-609.

23. Witham TF, Khavkin YA, Gallia GL, Wolinsky JP, Gokaslan ZL. Surgery insight: current management of epidural spinal cord compression from metastatic spine disease. Nat Clin Pract Neurol. 2006;2(2):87-94

24. Steinmetz MP Mekhail A, Benzel EC. Management of metastatic tumors of the spine: strategies and operative indications. Neurosurg Focus. 2001;11(6):e2.

25. Ecker RD, Endo T, Wetjen NM, Krauss WE. Diagnosis and treatment of vertebral column metastases. Mayo Clin Proc. 2005:80(9):1177-86.

26. Posner JB. Neurologic complications of cancer. Philadelphia: Fa Davis; 1995.

27. Helweg-Larsen S, Sørensen PS. Symptoms and signs in metastatic spinal cord compression: a study of progression from first symptom until diagnosis in 153 patients. Eur $\mathrm{J}$ Cancer. 1994:30A(3):396-8.

28. Greenberg HS, Kim JH, Posner JB. Epidural spinal cord compression from metastatic tumor: results with a new treatment protocol. Ann Neurol. 19808(4):361-6.

29. Gilbert RW, Kim JH, Posner JB. Epidural spinal cord compression from metastatic tumor: diagnosis and treatment. Ann Neurol. 1978;3(1):40-51.

30. Onimus M, Papin P, Gangloff S. Results of surgical treatment of spinal thoracic and lumbar metastases. Eur Spine J. 1996;5(6):407-11.

31. Schick U, Marquardt G, Lorenz R. Intradural and extradural spinal metastases. Neurosurg Rev. $2001 ; 24(1): 1-5$.

32. Schiff D, O'Neill BP, Wang CH, O'Fallon JR. Neuroimaging and treatment implications of patients with multiple epidural spinal metastases. Cancer. 1998;83(8):1593-601.

33. Livingston KE, Perrin RG. The neurosurgical management of spinal metastases causing cord and cauda equina compression. J Neurosurg. 1978;49(6):839-43 